\title{
高熵过渡金属碳化物陶瓷的研究进展
}

\author{
王皓轩 ${ }^{1}$, 刘巧沐 ${ }^{2}$, 王一光 ${ }^{3}$
}

(1. 西北工业大学 超高温结构复合材料重点实验室, 西安 710072; 2. 中国燃气涡轮研究院, 成都 610500; 3. 北京 理工大学 先进技术结构研究院, 北京 100081)

摘 要: 高熵陶瓷作为新型材料, 较大的构型熵赋予其独特的性能, 其中高熵过渡金属碳化物有望成为高超声速飞行 器热防护系统的备选材料。相比于单组元碳化物陶瓷, 高熵化的单相陶瓷在综合性能上有较大地提高。目前, 高熵过 渡金属碳化物陶瓷的研究还处于初始阶段, 关于高熵过渡金属碳化物的成分设计和理论分析还缺少足够的研究支撑。 另外，如何制备高纯度高熵过渡金属碳化物还需要进一步探索。在高熵过渡金属碳化物陶瓷的性能方面，还缺少深入 的研究。本文针对高熵陶瓷的理论设计和制备方法展开综述, 详细介绍了高熵过渡金属碳化物的力学、热导及抗氧化 性能的研究进展, 并指出了高熵过渡金属碳化物陶瓷在超高温陶瓷领域存在的科学问题, 展望了高熵过渡金属碳化 物陶瓷未来的发展方向。

关 键 词: 高熵过渡金属碳化物陶瓷; 构型熵; 力学性能; 热导性能; 抗氧化性能; 综述

中图分类号: TQ174 文献标识码: A

\section{Research Progress of High-entropy Transition Metal Carbide Ceramics}

\author{
WANG Haoxuan ${ }^{1}$, LIU Qiaomu ${ }^{2}$, WANG Yiguang ${ }^{3}$ \\ (1. Science and Technology on Thermostructural Composite Materials Laboratory, Northwestern Polytechnical University, \\ Xi'an 710072, China; 2. China Gas Turbine Establishment, Chengdu 610500, China; 3. Institute of Advanced Structure \\ Technology, Beijing Institute of Technology, Beijing 100081, China)
}

\begin{abstract}
As a new emergence material, high-entropy ceramics possess unique properties due to its high configurational entropy. Among these ceramics, high-entropy transition metal carbide ceramics (HETMCC) are expected to be the potential candidates for the thermal protection system of hypersonic aircraft. Compared with single-component ceramics, the comprehensive performance of single-phase HETMCC is greatly improved. At present, the research on HETMCC is still in the initial stage, and the composition design and theoretical analysis of HETMCC are lack of sufficient research support. In addition, it is necessary to further explore the preparation of high purity HETMCC. In terms of the properties of HETMCC, further in-depth research has been conducted. In this paper, the theoretical design and preparation methods of high-entropy ceramics are reviewed. Research progress of the mechanical properties, thermal conductivity, and oxidation resistance properties of HETMCC are introduced in detail. The concerned scientific issues of HETMCC are pointed out and their future development direction are also prospected.
\end{abstract}

Key words: high-entropy transition metal carbide; configurational entropy; mechanical property; thermal conductivity; oxidation-resistance; review

收稿日期: 2020-07-02; 收到修改稿日期：2020-09-27; 网络出版日期：2020-10-19

基金项目: 国家自然科学基金(51972027) National Natural Science Foundation of China (51972027)

作者简介: 王皓轩(1994-), 男, 博士研究生. E-mail: wanghaoxuan@mail.nwpu.edu.cn WANG Haoxuan(1994-), male, PhD candidate. E-mail: wanghaoxuan@mail.nwpu.edu.cn

通信作者: 王一光，教授. E-mail: wangyiguang@bit.edu.cn WANG Yiguang, professor. E-mail: wangyiguang@bit.edu.cn 
2004 年，叶均蔚和 Murty 首先提出了高熵合金 (High Entropy Alloys, HEA)的概念。高摘合金也叫 多组元合金，最初认为是由 5 种或 5 种以上的合金 元素组成，且每种元素的含量在 $5 \%$ 35\%之间的多 组元材料体系 ${ }^{[1-2]}$ 。随着对合金研究的深入, 4 种元 素的合金体系也被认为是高熵合金 ${ }^{[3-4]}$ 。与传统合金 组分处于相图的边际不同, 高熵合金的组元常集中 于多元相图的中心区域, 因此它的出现极大地丰富 了合金材料体系。高熵合金具有热力学上的高熵效 应、结构上的晶格畸变效应、动力学上的迟滞扩散 效应以及性能上的鸡尾酒效应, 使其具有较高的硬 度和强度、较好的耐磨性以及优异的抗腐蚀性等, 这些优异的性能引起了材料工作者的广泛关注 ${ }^{[5-6]}$ 。 将此概念推广到陶瓷领域, 可以把等摩尔或接近等 摩尔比的不少于四种元素的阳离子或阴离子组成 的单相化合物称为高熵陶瓷(High Entropy Ceramics, $\mathrm{HEC})^{[7-9]}$ 。可以预料, 由于 “鸡尾酒” 效应, HEC 与 HEA 一样, 有望表现出优异的机械和物化性能 ${ }^{[10-17]}$ 。 2015 年, Rost 等 ${ }^{[18]}$ 首先合成了高熵氧化物 $(\mathrm{MgNiZnCuCo}) \mathrm{O}$, 这是首次发表的有关 HEC 的论 文, 自此掀起了 HEC 的研究热潮。目前, 已经合成 了多种具有不同晶体结构的 HEC 体系, 如图 1 所 示 ${ }^{[19-30]}$ 。这些 HEC 比单组元陶瓷具有更好的机械性 能 ${ }^{[31]}$ 、离子电导率 ${ }^{[32]}$ 、耐腐蚀性 ${ }^{[33-36]}$ 、核废料固化 能力 ${ }^{[37]}$ 和低温电导率 ${ }^{[38]}$ 等。

目前, 研究最多的 HEC 体系是氧化物体系, 对 非氧化物陶瓷体系的研究相对较少。非氧化物陶瓷, 特别是难熔金属碳化物和硼化物陶瓷, 由于其较高 的熔点、优异的高温强度、较好的热稳定性以及相 对较低的热膨胀系数等特点, 在高超声速飞行器热

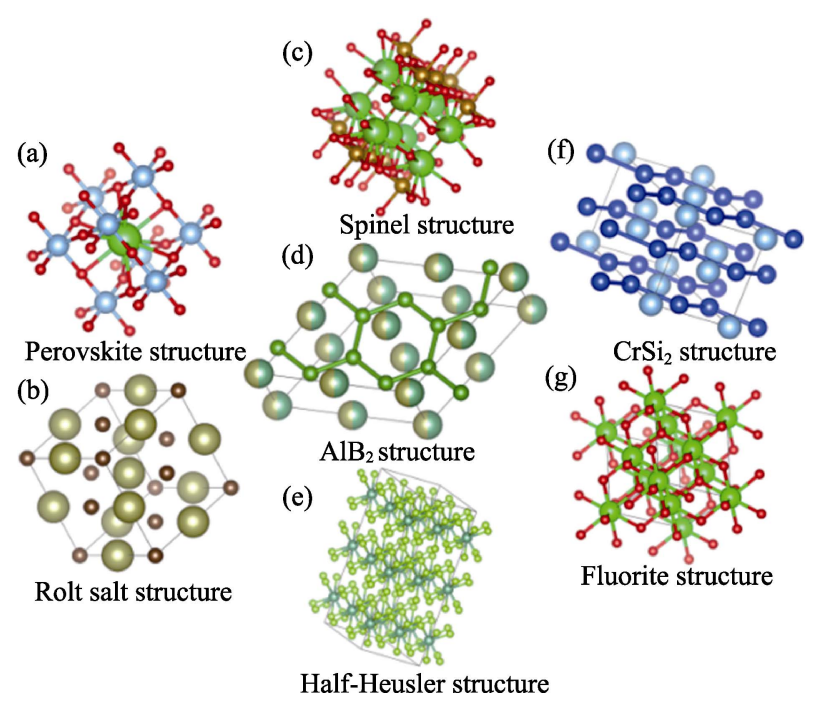

图 1 晶体结构示意图 ${ }^{[19-30]}$

Fig. 1 Schematic diagrams of crystal structure ${ }^{[19-30]}$
防护系统(Thermal Protection System, TPS)中有着关 键应用 ${ }^{[39-41]}$ 。这类陶瓷又称为超高温陶瓷(Ultra High Temperature Ceramics, UHTCs), 主要包括 $\mathrm{ZrB}_{2}$ 、 $\mathrm{ZrC} 、 \mathrm{HfB}_{2}$ 和 $\mathrm{HfC}$ 等 ${ }^{[42-45]}$ 。UHTCs 的高温快速氧化 是其应用的瓶颈 ${ }^{[46-48]}$ 。尽管研究人员尝试通过添加 $\mathrm{SiC} 、 \mathrm{TaC} 、 \mathrm{~B}_{4} \mathrm{C}$ 和 $\mathrm{MoSi}_{2}$ 等物质来提高陶瓷的抗氧 化性 ${ }^{[99-60]}$, 但是当温度达到 $1600{ }^{\circ} \mathrm{C}$ 以上时, 掺杂物 对陶瓷氧化性能的改善作用仍然十分有限。为了进 一步满足高超声速飞行器的需求, 对抗氧化性能更优 的新型超高温陶瓷的开发备受关注。2016 年 Luo 等 ${ }^{[61]}$ 首次制备了一系列具有密排六方 $(\mathrm{HCP})$ 结构的高熵 嗍化物陶瓷, 其氧化速率比单组元嗍化物降低一个量 级。禇衍辉等 ${ }^{[62]}$ 则探索了 $\left(\mathrm{Hf}_{0.2} \mathrm{Zr}_{0.2} \mathrm{Ta}_{0.2} \mathrm{Nb}_{0.2} \mathrm{Ti}_{0.2}\right) \mathrm{C}$ 的抗氧化性能, 其氧化速率同样低于单组元碳化 物。超高温高摘陶瓷的出现有望解决单相陶瓷的高 温抗氧化性能较差的问题, 为 UHTCs 的研究提供 了新的思路。

为此, 本文将以高熵过渡金属碳化物陶瓷(Highentropy Transition Metal Carbide Ceramics, HETMCC) 为对象, 简述 HETMCC 的合成理论和制备方法, 针 对超高温领域关注的力学性能和热导性能进行综述, 并就其抗氧化性能及改性展开分析讨论。

\section{1 高熵过渡金属碳化物的设计}

早期, HEC 的制备采用随机选取的方法对几种 化合物或者单质进行组合, 通过特定的工艺对混合 物进行烧结。但是这种方法试验量巨大、实验周期 长, 对原材料的消耗较大, 并且无法保证合成物为 单相固溶体。为了更精确地制备单相固溶体, 开发 新的 HEC 材料体系, 研究人员尝试通过模拟计算的 方式, 预测 HEC 体系存在的可能性, 同时分析晶体 结构稳定性。

HEC 单相形成能力通常以吉布斯自由能为判 据。对于陶瓷材料, 混合前后体系的自由能变化采 用以下公式进行计算 ${ }^{[63-65]}$ :

$$
\Delta G_{\text {mix }}=\Delta H_{\text {mix }}-T \Delta S_{\text {mix }}
$$

其中, $\Delta G_{\text {mix }}$ 为混合吉布斯自由能, $\Delta H_{\text {mix }}$ 为混合焓, $T$ 为温度, $\Delta S_{\text {mix }}$ 为混合熵。在 HEC 中, $\Delta S_{\text {mix }}$ 通常用构 型熵变化 $\Delta S_{\text {conf }}$ 来表示。根据玻尔兹曼公式, 对于高 熵材料体系, 系统的构型熵表述为:

$$
\Delta S_{\text {conf }}=-R \sum_{i=1}^{n} c_{i} \ln c_{i}
$$

其中, $R$ 为气体常数, $c_{i}$ 为组成元素 $i$ 的摩尔分数, $n$ 为材料的组成元素数, 组元越多 $S_{\mathrm{conf}}$ 越高。对于给 
定数量的组元，上述公式可简化为:

$$
\Delta S_{\text {conf }}=-R(1 / n \ln 1 / n+\cdots+1 / n \ln 1 / n)=R \ln n
$$

当 $\mathrm{HEC}$ 的组元为等摩尔比时，构型熵达到最大 值。除上述计算方法预测 HEC 存在的可能性之外, 还可以使用计算相图(Calculation of Phase Diagrams, CALPHAD) 来判定 ${ }^{[66-67]}$, 即通过热力学数据库, 使 用数值插值或外推法来计算相图并预测新的高熵材 料稳定相的组成。通过相图计算的方式, 研究人员 揭示了部分轻质高熵合金 ${ }^{\left[{ }^{[8]}\right.}$ 相的形成规律。但是该 方法存在一定的局限性, 当高熵材料中元素摩尔比 接近时, CALPHAD 预测结果可靠性将大幅度降低。

对于 HEC 的晶体结构预测, 通常采用基于密度 泛函理论(Density Functional Theory, DFT) ${ }^{[69-70]}$ 的特 定准随机结构(Special Quasirandom Structures, SQS) 和相干势近似(Coherent Potential Approximation, CPA)两种方法进行描述 ${ }^{[71-73]}$ 。SQS 主要描述高熵材 料最优的周期性结构, 并且固溶体的原子排布和晶 体结构接近于理想状态。在 SQS 方法指导下，人们 成功合成了单相 HETMCC $\left(\mathrm{Zr}_{0.25} \mathrm{Nb}_{0.25} \mathrm{Ti}_{0.25} \mathrm{~V}_{0.25}\right) \mathrm{C}^{[74]}$ 。 虽然 SQS 具有一定的指导作用，但是在实际应用中， 该方法需要构建多个理想模型, 耗费大量的计算成 本。CPA 是一种计算平均场的方法, 模型中包含了 所有原子, 不考虑高熵材料中晶格畸变带来的影 响。与 SQS 方法相比, CPA 的计算成本较低。上述 方法对高熵材料体系具有普适性。

对于 HETMCC 材料, 还可以通过熵形成能力 (Entropy Forming Ability, EFA)作为其稳定性的判 据 ${ }^{[31]}$ 。通过测量材料的能量分布谱来判断能否形成 单相 HETMCC。窄的能量分布谱意味着达到亚稳态 构型只需要消耗少量能量, 在有限的温度下具有较 高的系统随机性。相反, 宽的能量分布谱意味着引 入不同的构型会有高的能量势垒, 导致形成第二 相。在有限温度下, HETMCC 的 EFA 值可通过以下 公式进行描述:

$$
\begin{gathered}
\mathrm{EFA}=\left(\sqrt{\sum_{i=1}^{n} g_{i}\left(H_{i}-H_{\text {mix }}\right)^{2} /\left[\left(\sum_{i=1}^{n} g_{i}\right)-1\right]}\right)^{-1} \\
H_{\text {mix }}=\sum_{i=1}^{n} g_{i} H_{i} / \sum_{i=1}^{n} g_{i}
\end{gathered}
$$

其中, $n$ 是样品的组元数, $g_{i}$ 为兼并值, $H_{\text {mix }}$ 是混合 焓, 其值接近于样品构型焓 $H_{i}$ 的平均值。Sarker 等 ${ }^{[31]}$ 用 EFA 方法计算出 56 个五元碳化物结构的能 量分布谱。对于 $\mathrm{MoNbTaVWC}_{5} 、 \mathrm{HfNbTaTiZrC}_{5}$ 、 $\mathrm{HfNbTaTiVC}_{5}$ 三种碳化物, 它们具有较大的 EFA 值 $\left.(\text { 均>100 (eV/atom })^{-1}\right)$, 表明其能量势垒较小, 因此
倾向于形成单相 HETMCC。而对于 HfMoTaWZrC 5 、 $\mathrm{HfMoTiWZrC}_{5}$ 和 $\mathrm{HfMoVWZrC}_{5}$, 其 EFA 值从 45 降 到 $37(\mathrm{eV} / \text { atom })^{-1}$, 表明在形成这三种碳化物时可能 会产生第二相。据此判断 HETMCC 的合成阈值约 为 $50(\mathrm{eV} / \text { atom })^{-1}$ 。为了验证理论计算的正确性, 在 $2200{ }^{\circ} \mathrm{C}$ 下合成了 9 种碳化物陶瓷, 发现具有较大 EFA 值的 HETMCC 均形成了单相固溶体, 而 EFA 值较小的陶瓷出现了第二相, 实验结果与计算预测 符合。该方法为研究人员设计 HETMCC 提供了系 统的指导。

\section{2 高熵过渡金属碳化物的制备}

HETMCC 制备的研究对象主要涉及薄膜、粉体 以及块体材料。

\section{1 高熵涂层的制备}

HETMCC 薄膜主要采用磁控溅射工艺进行制 备, 可以将五种金属元素分别作为阴极靶材, 采用 $\mathrm{CH}_{4}$ 作为反应气体，在溅射沉积的同时使原子相互 反应, 这样可以获得成分均匀并且结构稳定的纳米 级(TiZrNbHfTa)C 高熵涂层 ${ }^{[75]}$ 。还能直接以高熵合 金 AlCrTaTiZr 和石墨作为阴极靶材, 在氩气和氮 气气氛中, 运用磁控溅射的方法在硅基板上制备 出具有致密柱状结构的复合高熵(AlCrTaTiZr) $\mathrm{NC}_{y}$ 涂层 ${ }^{[76]}$ 。

\section{2 高熵粉体的制备}

HETMCC 粉体制备的方法主要有固相反应法

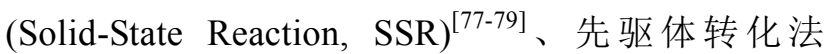
(Polymer Derived Gramic Technique, PDCT) ${ }^{[80]}$ 和熔

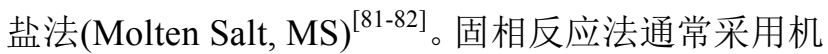
械合金化(Mechanical Alloying, MA)制备粉体，所用 粉体为单组元碳化物或者氧化物粉体。以不同种类 的碳化物粉体为原料, 酒精为介质, 经湿法球磨给 予粉体较高的能量后，可以获得等摩尔比的非晶态 碳化物混合粉体。再在 $1950{ }^{\circ} \mathrm{C}$ 对过篮后的粒径均 匀的混合粉体进行烧结, 依靠高温下各元素的相互 扩散获得 HETMCC 粉体 ${ }^{[77]}$ 。而氧化物粉体目前通 常采用一步法和两步法结合碳热还原的方式进行处 理 ${ }^{[79-80]}$ 。首先将金属氧化物和石墨混合，然后对粉 体进行球磨，再把混合粉体压成圆盘，通过固态碳 热还原法在 $1600{ }^{\circ} \mathrm{C}$ 下获得纯净的混合碳化物粉体, 最后经过 $2000{ }^{\circ} \mathrm{C}$ 热处理实现碳化物粉体的固溶反 应 ${ }^{[78]}$ 。虽然两步法可以获得 HETMCC 粉体, 但是制 备时间较长, 并且含有一定量的氧元素。褚衍辉等 ${ }^{[79]}$ 直接将球磨后的混合粉体放入石墨坩埚中, 在真空 
炉中加热至所需温度, 经过一定时间保温后可以实 现一步碳热法制备高纯 $\left(\mathrm{Zr}_{0.25} \mathrm{Ta}_{0.25} \mathrm{Nb}_{0.25} \mathrm{Ti}_{0.25}\right) \mathrm{C}$, 大 大提高了 HETMCC 粉体的制备效率。

先驱体转化法可用来制备先驱体粉末。将过渡 族金属氯化物与乙醇以及其他化学试剂进行混合, 充分搅拌后获得 HETMCC 的先驱体溶液; 经过适 当温度的聚合反应, 冷却后获得 HETMCC 的先驱 体固相聚合物, 在此过程中, 分子可以充分扩散; 最后经过高温热处理, 先驱体发生分解和化学反 应, 形成具有单一结构的 HETMCC 粉体。禇衍辉 等 ${ }^{[80]}$ 将四种不同的氯化物与乙酰甲烷置于乙醇中 溶解, 再经过 $200{ }^{\circ} \mathrm{C}$ 聚合反应和 $2000{ }^{\circ} \mathrm{C}$ 高温热处 理 $1 \mathrm{~h}$ 成功制备了平均粒径为 $800 \mathrm{~nm}$ 的超细 $\left(\mathrm{Hf}_{0.25} \mathrm{Nb}_{0.25} \mathrm{Zr}_{0.25} \mathrm{Ti}_{0.25}\right) \mathrm{C}$ 纳米粉体。

熔盐法则是采用数种低熔点的盐类作为反应介 质, 将反应物溶解在熔盐中进行反应。与固相粉体 中原子相互扩散的速率相比, 高温下熔融状态的盐 类物质原子扩散速率明显加快, 可以缩短粉体的制 备时间。充分反应后采用适当溶剂溶解并洗涤盐类 物质, 可以获得 HETMCC 粉体。该方法工艺简单, 合成温度低, 获得的粉体成分均匀。褚衍辉等 ${ }^{[81]}$ 以四种金属粉体、碳粉为原料, 采用氯化钾作为熔 盐介质, 在第一性原理指导下 $1300^{\circ} \mathrm{C}$ 合成了具有单 相岩盐结构的纳米 $\left(\mathrm{Ta}_{0.25} \mathrm{Nb}_{0.25} \mathrm{Ti}_{0.25} \mathrm{~V}_{0.25}\right) \mathrm{C}$ 粉体, 该 粉体的组成均匀性较好。这是首次利用熔盐法制备 HETMCC 粉体的报道。Jagadeesh 等 ${ }^{[82]}$ 把氧化物与 石墨混合物压实成圆柱形圆盘, 热处理后得到足够 强度的块体。再以圆盘为阴极, 石墨为阳极, 用熔融 氯化钻作为电解质, 经过短时间的电解, 在阴极制备 出纳米 HETMCC 粉体。

\section{3 高熵块体的制备}

对于 HETMCC 块体, 目前主要通过热压(Hot Pressed Sintering, HP)、放电等离子体(Spark Plasma Sintering, SPS) 以及反应闪烧放电等离子体(Reactive Flash Spark Plasma Sintering, RFSPS)等方法对上述 碳化物粉体进行烧结。采用 HP 工艺制备 HETMCC, 得到的材料气孔较多, 制备时间较长 ${ }^{[73]}$; 采用 SPS 制备 HETMCC, 在缩短制备时间的同时, 可以有效 降低 HETMCC 的气孔率 ${ }^{[33]}$; 采用 RFSPS 不仅可以 降低烧结温度, 而且能缩短陶瓷的制备周期, 提高 陶瓷的相对密度 ${ }^{[28]}$ 。

然而, 目前高熵陶瓷的制备方法还存在一些不 利因素: (1)由于机械合金化方法的引入, 球磨后的 粉体通常包含由磨球以及球磨罐带来的杂质元素, 并且烧结后的陶瓷容易出现微区元素分布不均匀的
情况; (2)高熵陶瓷的迟滞扩散效应使得 HETMCC 的致密化较为困难, 获得的陶瓷块体会含有少量的 气孔, 不利于后续性能的测试; (3)采用氧化物进行 碳化反应时, 陶瓷内会残留少量的氧元素。因此为 了提高烧结后陶瓷的相对密度以及减少杂质元素含 量, 改进陶瓷粉体的合成方法、研究新的烧结工艺 有望成为未来重点研究方向。

\section{3 高熵过渡金属碳化物陶瓷的性能}

\section{1 力学性能}

高熵效应导致陶瓷材料性能的变化, 特别是 力学性能的改变是陶瓷工作者关注的核心之一。通 过研究碳含量对高熵( TiAlCrNbY)C 薄膜硬度的影 响 ${ }^{[33]}$, 可以发现碳原子含量为 $69 \%$ 左右时, 得到的 薄膜硬度可达 $(22.6 \pm 1.2) \mathrm{GPa}$ 。而对于不同碳含量的 $(\mathrm{CrNbSiTiZr}) \mathrm{C}_{x}$ 薄膜 ${ }^{[84]}$ 的研究发现, 当甲烷流量占 比为 $3 \%$ 时, 所获得的薄膜硬度最大, 可达 $32.8 \mathrm{GPa}$, 远高于采用混合法则计算的 $23.4 \mathrm{GPa}$, 如图 2(a)所 示。这归因于高熵薄膜的固溶强化机制, 薄膜中元 素种类的增加使得材料构型熵增大, 晶格畸变加剧, 大幅度提高了材料的硬度。受到固溶强化机制的启发,
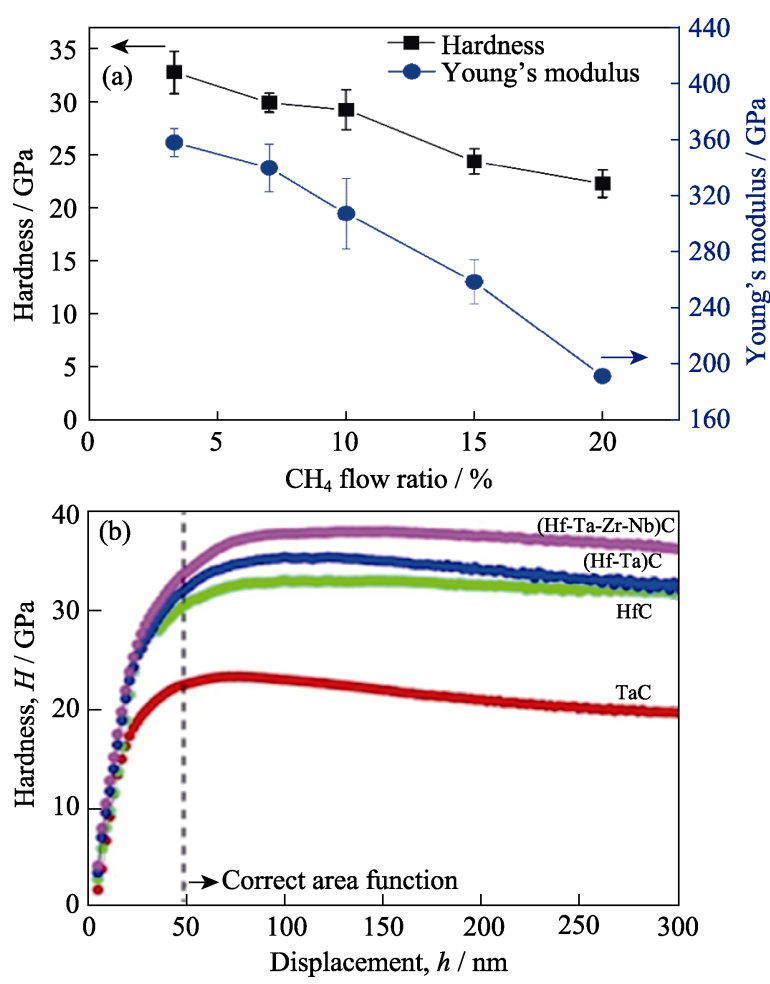

图 2 (a)不同碳含量高熵 $\mathrm{CrNbSiTiZrC}_{x}$ 薄膜硬度 ${ }^{[84]}$, (b) 不同 碳化物硬度-深度曲线 ${ }^{[87]}$

Fig. 2 (a) Hardness of the $\mathrm{CrNbSiTiZrC}_{x}$ with different carbon contents ${ }^{[84]}$, and (b) hardness depth-profles of the individual, binary and high-entropy carbide ${ }^{[87]}$ 


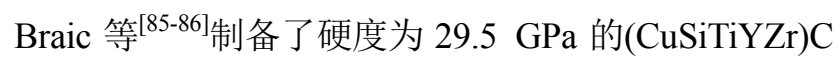
和 $30 \mathrm{GPa}$ 的 $(\mathrm{CrCuNbTiY}) \mathrm{C}$ HETMCC 薄膜, 所得薄 膜的硬度均高于混合法则的计算值。

通过测试 $\left(\mathrm{Zr}_{0.25} \mathrm{Nb}_{0.25} \mathrm{Ti}_{0.25} \mathrm{~V}_{0.25}\right) \mathrm{C}^{[74]}$ 的纳米压痕 硬度, 发现其值为(30.3 \pm 0.7$) \mathrm{GPa}$, 明显高于混合法 则计算得到的 $25 \mathrm{GPa}$ 。采用同样的方法测试 HfNbTaTiZrC ${ }_{5}^{[31]}$ 的硬度和弹性模量, 与混合法则预 测值相比, 分别超出预测值的 $10 \%$ 和 $50 \%$ 。图 2(b) 为 Csanadi 等 ${ }^{[87]}$ 研究的不同碳化物硬度-深度曲线, 对比发现高熵化后的碳化物陶瓷硬度有较大提升。 为了进一步比较块体高熵陶瓷与单组元陶瓷的硬度, 我们对部分数据进行归纳, 具体如表 1 所示。通过 对比陶瓷的硬度值可以发现, 与混合法则预测值以 及单组元陶瓷的硬度相比较, HETMCC 的硬度都有 所增加, 并且大部分陶瓷高熵化以后提升较大。固 溶强化效应对 HETMCC 硬度的提升十分明显。

张程爆等 ${ }^{[91]}$ 探索了 $(\mathrm{TaHfZrNb}) \mathrm{C}$ 在 $1400 \sim 1600{ }^{\circ} \mathrm{C}$ 和 150 300 MPa 的压缩应力下的蠕变行为。通过测 试发现高熵化以后的 HETMCC 的稳态蠕变比相应 的单相碳化物的蠕变速率减小了将近 $90 \%$ 。并且测 得该 HETMCC 应力指数 $n$ 在 2.34 2.89 的范围内, 平均活化能为 $212 \mathrm{~kJ} / \mathrm{mol}$ 。

综上, HETMCC 硬度的提升以及蠕变速率降低 的原因在于陶瓷引入了较多的组分, 不同阳离子的

表 1 部分碳化物陶瓷硬度 ${ }^{[31,74,88-90]}$

Table 1 Hardness of some carbide ceramics ${ }^{[31,74,88-90]}$

\begin{tabular}{lcc}
\hline \multicolumn{1}{c}{ HEC } & Hardness/GPa & Average/GPa \\
\hline $\mathrm{HfC}^{[31]}$ & 25 & - \\
$\mathrm{TaC}^{[31]}$ & 14 & - \\
$\mathrm{ZrC}^{[31]}$ & 24 & - \\
$\mathrm{TiC}^{[31]}$ & 31 & - \\
$\mathrm{NbC}^{[31]}$ & 17 & - \\
$\mathrm{WC}^{[88]}$ & 14 & - \\
$\mathrm{VC}^{[31]}$ & 29 & - \\
$\mathrm{Mo}_{2} \mathrm{C}^{[31]}$ & 27 & - \\
$(\mathrm{ZrNbTiV}) \mathrm{C}^{[74]}$ & 30 & 25 \\
$(\mathrm{HfTaZrNb}) \mathrm{C}^{[87]}$ & 36 & 20 \\
$(\mathrm{TiVNbTaW}) \mathrm{C}^{[31]}$ & 28 & 21 \\
$(\mathrm{TiHfTaWZr}) \mathrm{C}^{[31]}$ & 33 & 22 \\
$(\mathrm{TiHfNbTaMo}) \mathrm{C}^{[89]}$ & 27 & 23 \\
$(\mathrm{TiZrNbTaMo}) \mathrm{C}^{[90]}$ & 32 & 23 \\
$(\mathrm{VNbTaMoW}) \mathrm{C}^{[31]}$ & 27 & 20 \\
$(\mathrm{HfTaZrTiNb}) \mathrm{C}^{[31]}$ & 32 & 28 \\
$(\mathrm{TiZrHfTaW}) \mathrm{C}^{[89]}$ & 24 & 22 \\
$(\mathrm{TiHfNbTaW}) \mathrm{C}^{[31]}$ & 31 & 23 \\
$(\mathrm{TiHfVNbTa}) \mathrm{C}^{[31]}$ & 29 & \\
\hline
\end{tabular}

尺寸差异较大，形成固溶体时容易产生大量的晶格 畸变, 增大了位错运动的阻力, 导致在外力作用下, 原子滑移十分困难，限制塑性变形所必须的位错运 动。对于 HETMCC, 大部分固溶体为置换型固溶体, 溶质原子的引入引发了局部点阵畸变, 溶质原子与 位错产生的交互作用降低了位错周围的弹性应变 能。从宏观上来说, 这种作用增加了材料对外界压 力的抵抗能力, 导致材料变形需要做更多的机械 功。因此对于 HETMCC 陶瓷在硬度和抗蠕变方面 的改性将主要集中在元素调控, 通过调整晶胞内阳 离子的尺寸差异, 进而控制位错的分布, 实现 HETMCC 陶瓷力学性能的优化。

\section{2 热导性能}

HETMCC 较高的构型熵和由此产生的晶格畸 变会影响材料的热导性能, 因此, HETMCC 的热导 变化也是研究热点。 $\left(\mathrm{Hf}_{0.2} \mathrm{Zr}_{0.2} \mathrm{Ta}_{0.2} \mathrm{Nb}_{0.2} \mathrm{Ti}_{0.2}\right) \mathrm{C}$ 高摘 陶瓷 ${ }^{[22]}$ 在室温下的热导仅为 $6.45 \mathrm{~W} /(\mathrm{m} \cdot \mathrm{K})$, 远低于 $\mathrm{HfC}(29.3 \mathrm{~W} /(\mathrm{m} \cdot \mathrm{K}))$ 和 $\mathrm{TaC}(33.5 \mathrm{~W} /(\mathrm{m} \cdot \mathrm{K}))$ 。对于固体 材料, 声子和电子是传导热量的主要载体, 相比于 声子贡献达 $75 \%$ 的 $\mathrm{TaC}$, 高熵化以后的碳化物声子 贡献率发生了巨大改变。由于 5 种金属原子 $(\mathrm{Hf} 、 \mathrm{Zr}$ 、 $\mathrm{Ta} 、 \mathrm{Nb}$ 和 $\mathrm{Ti}$ ) 在阳离子位置上的尺寸和结合强度不 同, 使得 $\left(\mathrm{Hf}_{0.2} \mathrm{Zr}_{0.2} \mathrm{Ta}_{0.2} \mathrm{Nb}_{0.2} \mathrm{Ti}_{0.2}\right) \mathrm{C}$ 存在明显的原子 尺度上的晶格畸变。随着元素含量以及种类的增加, 晶格畸变不断加剧, 导致该碳化物陶瓷材料的热导 率下降。高摘氧化物中也存在相同现象 ${ }^{[18]}$ 。另外一 种 HETMCC 陶瓷 $\left(\mathrm{Zr}_{0.25} \mathrm{Nb}_{0.25} \mathrm{Ti}_{0.25} \mathrm{~V}_{0.25}\right) \mathrm{C}^{[74]}$ 在室温 下的热导率为 $(15.3 \pm 0.3) \mathrm{W} /(\mathrm{m} \cdot \mathrm{K})$, 热扩散率为 $(5.2 \pm 0.1) \mathrm{mm}^{2} / \mathrm{s}$, 所有值均小于单组元碳化物陶瓷。 通过测试多孔 $\left(\mathrm{Zr}_{0.2} \mathrm{Hf}_{0.2} \mathrm{Nb}_{0.2} \mathrm{Ta}_{0.2} \mathrm{Ti}_{0.2}\right) \mathrm{B}_{6}$ 和 $\left(\mathrm{Zr}_{0.2} \mathrm{Hf}_{0.2} \mathrm{Ti}_{0.2} \mathrm{Nb}_{0.2} \mathrm{Ta}_{0.2}\right) \mathrm{C}$ 陶瓷 ${ }^{[93-94]}$ 热导性能, 获得 它们的室温热导分别为 0.51 和 $0.39 \mathrm{~W} /(\mathrm{m} \cdot \mathrm{K})$, 均低 于多孔的单组元硼化物/碳化物陶瓷, 且在一定的温 度范围内均显示出较低的热导(图 3)。

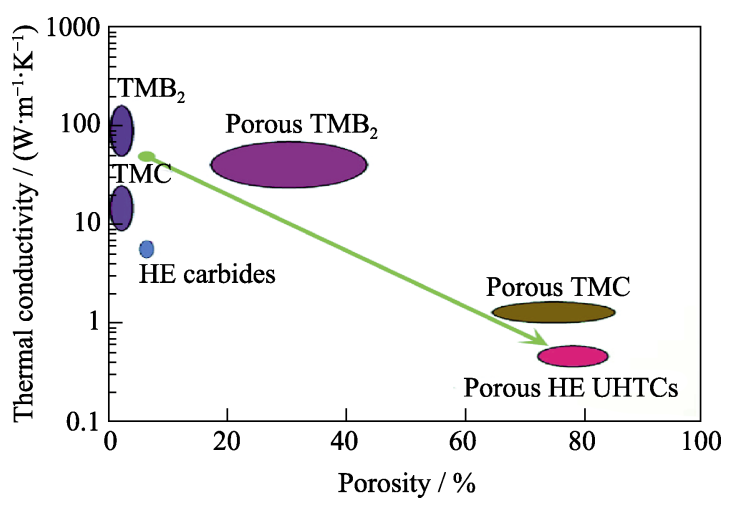

图 3 高熵陶瓷热导性能 ${ }^{[93]}$

Fig. 3 Thermal conductivities for high-entropy ceramics ${ }^{[93]}$ 
综上, HETMCC 热导降低主要是由以下原因造 成的: (1)材料内部存在较高的构型摘, 碳化物晶格 内不同原子存在质量和尺寸差异, 当晶格中的原子 被取代时, 晶格内部产生大量的晶格畸变, 增加了 声子散射几率, 从而导致材料的热导较低; (2)热导 性能的提高源于 HETMCC 内热阻的提高。由于原 子缺陷与热阻存在正相关性, 当原子发生置换时, 会产生质量和应力场的波动, 这种波动导致了热阻 的上升，从而降低热导; (3)在高摘陶瓷的制备过程 中, 会存在纳米尺度的析出相, 这种相的存在增加 了晶界的数量, 同样对声子的传导起到了阻碍作 用。因此, 为了进一步提高 HETMCC 的热导性能, 可以尝试在陶瓷内部引入较多的组元, 提高声子散 射在热导中的贡献率。与此同时, 适当提高原子尺 寸差异, 增加晶格内部的点阵畸变, 也可以提高声 子散射。上述方法将对后续研究 HETMCC 陶瓷的 热导性能提供参考思路。

\section{3 抗氧化性能}

UHTCs 在高温领域应用的一个关键性能是抗 氧化性能, 但是单组元难熔金属碳化物的高温抗氧化 性能有待进一步提高。在传统氧化的研究中, 褚衍 辉等 ${ }^{[95}$ 探索了 $\left(\mathrm{Hf}_{0.2} \mathrm{Zr}_{0.2} \mathrm{Ta}_{0.2} \mathrm{Nb}_{0.2} \mathrm{Ti}_{0.2}\right) \mathrm{C}$ 高熵碳化物 在 800 1200 ${ }^{\circ} \mathrm{C}$ 的抗氧化性, 如图 4(a)所示。与单组 元碳化物陶瓷相比, HETMCC 表现出优异的抗氧化 性, 但是当温度超过 $1000{ }^{\circ} \mathrm{C}$, 氧化增重规律发生变 化并且氧化层裂纹开始增大。褚衍辉等虽然发现材料 具有较好的抗氧化性, 但是研究还主要集中在产生 横向裂纹的原因分析上。对 $\left(\mathrm{Hf}_{0.2} \mathrm{Zr}_{0.2} \mathrm{Ta}_{0.2} \mathrm{Nb}_{0.2} \mathrm{Ti}_{0.2}\right) \mathrm{C}$ 在 1300 1500 ${ }^{\circ} \mathrm{C}$ 的抗氧化性能进一步研究, 发现 陶瓷在 $1500{ }^{\circ} \mathrm{C}$ 氧化 $1 \mathrm{~h}$ 后陶瓷出现失重现象, 表明 氧化层完全失效, 同时指出其氧化的主导过程是 由氧元素向内扩散控制 ${ }^{[96]}$ 。王一光等 ${ }^{[97]}$ 也研究了 $\left(\mathrm{Hf}_{0.2} \mathrm{Zr}_{0.2} \mathrm{Ta}_{0.2} \mathrm{Nb}_{0.2} \mathrm{Ti}_{0.2}\right) \mathrm{C}$ 的高温抗氧化性, 当温 度超过 $1500{ }^{\circ} \mathrm{C}$, 短时间内的氧化可以观察到 $\mathrm{Ti}$ 元素的耗尽层, 随着氧化时间延长, 该耗尽层会 逐渐消失。据此, 王一光等 ${ }^{[97]}$ 认为 $\mathrm{HETMCC}$ $\left(\mathrm{Hf}_{0.2} \mathrm{Zr}_{0.2} \mathrm{Ta}_{0.2} \mathrm{Nb}_{0.2} \mathrm{Ti}_{0.2}\right) \mathrm{C}$ 的氧化是由含钛元素化合 物向外扩散控制的过程。在高温条件下, $\mathrm{TiC}$ 较其它 元素碳化物更容易发生活性氧化生成 $\mathrm{TiO}$ 气体, 并 向表层扩散，导致形成 $\mathrm{TiO}$ 耗尽层。另外，通过实验 可以发现，高温下 $\mathrm{Hf} 、 \mathrm{Zr} 、 \mathrm{Ti}$ 元素氧化较为显著，而 $\mathrm{Ta}$ 和 $\mathrm{Nb}$ 元素生成的氧化物逐渐减少, 这主要是由于 HETMCC 选择性氧化导致的 ${ }^{[97]}$, 这与 Backman 等 ${ }^{[98]}$ 研究结果相似。通过对第 $I V$ 副族和第 $V$ 副族元素在 高熵条件下元素氧化先后顺序的研究, 发现对氧
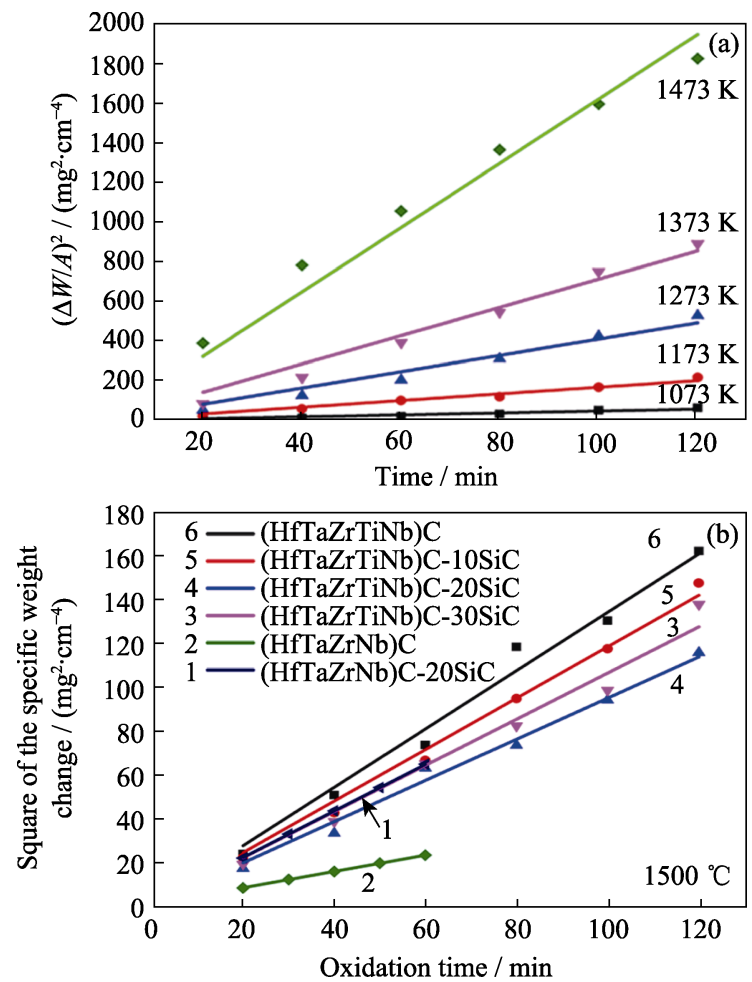

图 4 (a) $\left(\mathrm{Hf}_{0.2} \mathrm{Zr}_{0.2} \mathrm{Ta}_{0.2} \mathrm{Nb}_{0.2} \mathrm{Ti}_{0.2}\right) \mathrm{C}$ 在 $800 \sim 1200{ }^{\circ} \mathrm{C}$ 氧化增重 曲线 ${ }^{[95]}$, 和(b)不同体系碳化物在 $1500{ }^{\circ} \mathrm{C}$ 氧化增重曲线 ${ }^{[33,97,99]}$

Fig. 4 (a) Square of the specific weight change as a function of oxidation time at $800-1200{ }^{\circ} \mathrm{C}$ for $\left(\mathrm{Hf}_{0.2} \mathrm{Zr}_{0.2} \mathrm{Ta}_{0.2} \mathrm{Nb}_{0.2} \mathrm{Ti}_{0.2}\right) \mathrm{C}^{[95]}$, (b) square of the specific weight change as a function of oxidation time at $1500{ }^{\circ} \mathrm{C}$ for high-entropy carbide in different systems $^{[33,97,99]}$

原子抢夺能力表现为 $\mathrm{Hf}>\mathrm{Zr}>\mathrm{Ti}>\mathrm{Ta}>\mathrm{Nb}$ 。根据相似 相溶原理, Hf、 Zr、Ti 对应的稳定氧化物形成固溶 相 $\left(\mathrm{Hf}_{0.5} \mathrm{Zr}_{0.5}\right) \mathrm{TiO}_{4}, \mathrm{Ta}$ 和 $\mathrm{Nb}$ 则形成 $(\mathrm{Ta}, \mathrm{Nb}) \mathrm{O}_{5}$ 。正是 钛酸盐的形成有效保护了陶瓷基体，但是 $\mathrm{TiO}$ 向外 扩散时会留下许多孔洞，不利于 HETMCC 长时间 的抗氧化测试。为了进一步改善 HETMCC 抗氧化 性能，掺杂 $\mathrm{SiC}$ 对 HETMCC 进行改性，同样观察到 了 $\mathrm{Ti}$ 的耗尽层 ${ }^{[33]}$ 。另外, 由于生成 $\mathrm{SiO}$ 在最外层氧 化层形成了复合盐类物质 $\left(\mathrm{Hf}_{0.5} \mathrm{Zr}_{0.5}\right) \mathrm{TiO}_{4}$ 和 $\left(\mathrm{Hf}_{0.5} \mathrm{Zr}_{0.5}\right) \mathrm{SiO}_{4}$, 更加有效地阻碍了氧气的进入。但 是 $\mathrm{Si}$ 元素的存在使得 HETMCC 内的组元对于氧气 的争夺更加激烈，进一步抑制了 $\mathrm{Ta}$ 和 $\mathrm{Nb}$ 的扩散。 由于 HETMCC 中元素对于氧气存在不同的响应, 为了提高氧化层的致密性, 需要对 HETMCC 进行 元素调控。考虑到 $\mathrm{Ti}$ 对氧化层的破坏作用以及硅酸 盐对基体的保护作用，发展了四元 HETMCC $\left(\mathrm{Hf}_{0.25} \mathrm{Ta}_{0.25} \mathrm{Zr}_{0.25} \mathrm{Nb}_{0.25}\right) \mathrm{C}$ 及 $\left(\mathrm{Hf}_{0.25} \mathrm{Ta}_{0.25} \mathrm{Zr}_{0.25} \mathrm{Nb}_{0.25}\right) \mathrm{C}$ $\mathrm{SiC}^{[99]}$ 体系。图 4(b)总结了几种高熵过渡金属碳化物 陶瓷氧化增重曲线。在缺少 $\mathrm{Ti}$ 的情况下, 对于 $\mathrm{Ta}$ 和 $\mathrm{Nb}$ 的抑制扩散作用减缓, $\mathrm{Hf}_{6} \mathrm{Ta}_{2} \mathrm{O}_{17}$ 和 $\mathrm{Zr}_{6} \mathrm{Nb}_{2} \mathrm{O}_{17}$ 开始出现, 生成物均为 $\mathrm{A}_{6} \mathrm{~B}_{2} \mathrm{O}_{17}$ 相, $\mathrm{Hf}$ 与 $\mathrm{Zr}$ 以及 $\mathrm{Ta}$ 
和 $\mathrm{Nb}$ 分别进行 $\mathrm{A}$ 位和 $\mathrm{B}$ 位替换，这一点与 Backman 等 ${ }^{[98]}$ 氧化结果一致。由于缺少 $\mathrm{Ti}$ 元素对氧化层的破 坏, 陶瓷形成了相对致密的氧化层, 但是也造成 $\mathrm{CO}$ 气体难以排出, 在 HETMCC 的氧化层内产生大量 应力。由于材料尖端部位容易出现应力集中, 因此 陶瓷的棱角处会最早产生裂纹。引进 $\mathrm{Si}$ 元素会使 $\mathrm{CO}$ 无法聚集，为应力的释放提供了路径。

综上, 基于 HETMCC 选择性氧化的特点, 元素 调控显得尤为重要 ${ }^{[97-99]}$ 。高温下 $\mathrm{Hf} 、 \mathrm{Zr} 、 \mathrm{Ti}$ 和 $\mathrm{Si}$ 的存在对于 $\mathrm{Ta}$ 和 $\mathrm{Nb}$ 向外扩散的抑制作用较为明显, 选择元素时, 需要考虑元素的相互作用。Ti 虽然促 进最外致密氧化层的形成, 但是 $\mathrm{TiO}$ 的存在会导致 大量孔洞的产生。选择不含气态生成物的元素, 长 时间氧化容易造成应力集中，使氧化层产生贯穿性 裂纹。Si 可以很好地消除上述影响, 但在更高温度 下， $\mathrm{Si}$ 的氧化容易产生大量挥发性气体。因此, HETMCC 需要进一步优化设计。同时,由于 HETMCC 迟滞扩散效应使得难熔金属元素在陶瓷中的扩散变 得十分困难, 利用高熵化的性质可以进一步改善难 熔金属碳化物的抗氧化腐蚀性能。另外, 在 HETMCC 氧化的过程中, 可以发现并不是固溶的组元越多越 好，在利用陶瓷高熵效应的同时还需要考虑元素对 HETMCC 抗氧化性能的影响。如何在保证氧化层完 整性的前提下避免由于 HETMCC 氧化产生气体引 起的应力集中将成为未来重点研究方向。

\section{4 其他性能}

除上述性能外, 科研工作者还探索了 HETMCC 其他性能。研究发现碳含量较高的(TiZrNbHfTa)C 薄膜 ${ }^{[100]}$ 通过降低开路电位、腐蚀电流密度和腐蚀电 位, 表现出较好的抗电化学腐蚀性能。在碳化物薄 膜中, 孔隙率随碳含量的增加而降低, 碳含量高的 薄膜有较低的孔隙率, 有助于抑制腐蚀溶液的侵 蚀。在生物相容性测试中, 粘附在 HETMCC 薄膜上 活性细胞较多，附着细胞形态良好，并且存活时间 较长, 对细胞无有害刺激性作用, 说明 HETMCC 薄 膜还具有较好的生物相容性。

通过测试(TiNbTaZrHf)C ${ }^{[82]}$ 的电化学性能, 发 现其在超级电容器电极中具有潜在应用。电化学初 步评估包括记录循环伏安曲线、测量充放电曲线和 循环曲线。当充放电速度为 $10 、 20 、 30 、 50$ 和 $100 \mathrm{mV} / \mathrm{s}$ 时，比容量分别为 $95.2 、 71.0 、 58.1 、 42.2$ 和 $27.4 \mathrm{~F} / \mathrm{g}$, 经过 2000 个完整循环后, 容量保留率 为 $87 \%$ 。

在 25、300 和 $500{ }^{\circ} \mathrm{C}$ 对 HETMCC (ZrTaNbTi)C 进行抗辐照性能测试, 发现采用 $20 \mathrm{dpa}$ 剂量的
$3 \mathrm{MeV} \mathrm{Zr}$ 离子辐照后, (ZrTaNbTi)C 仍能保持较高的 相稳定性 ${ }^{[101]}$ 。根据纳米压痕测试推测出辐射引起的 硬度增大可能是由于位错环作为纳米压痕测试过程 中滑移的障碍以及晶格引起的应变导致的。该陶瓷 的出现有望成为核领域的新型结构材料。

\section{4 总结与展望}

HEC 的出现为陶瓷材料性能的提升带来了新 的可能, 目前已经对氧化物、过渡族金属碳化物和 硼化物等 HEC 展开了研究。较高的构型摘赋予 HEC 独特的性能, 各种具有均质单相结构的 HEC 表现出 优异的性能。其中关于 HETMCC 陶瓷的研究已有 初步的结果: (1)HETMCC 由于制备方法的限制, 通 常会引入少量杂质元素, 并且烧结时会产生少量的 微气孔, 因此发展 HETMCC 新的制备方法至关重 要; (2)由于 HETMCC 中引入了较多的组元, 导致晶 胞内存在原子尺寸差异, 产生了大量的晶格畸变, 使得位错运动阻力增大, 致使外力作用下滑移变得 十分困难, 较大程度地提升了陶瓷的硬度; (3)对于 高熵化的碳化物陶瓷，声子在热导中贡献率增加, 晶格畸变限制了声子的运动, 从而提高了 HETMCC 的热导性能，同时陶瓷内缺陷的增加引起的热阻变 化，进一步改善了陶瓷的热导性能; (4)HETMCC 的 扩散迟滞效应阻碍了介质对陶瓷基体的腐蚀作用。 此外, 由于 HETMCC 中存在 C 元素, 在氧化过程中 会产生大量气体，造成应力。不同元素对氧原子有 不同的响应, 因此, 还需要考虑 HETMCC 中元素的 调控。

目前, HETMCC 的研究还处于起步阶段, 今后 对于 HETMCC 关注的问题将主要集中以下几点:

1)关于 HETMCC 的成分设计还缺少足够的理 论支撑，对于是否形成高熵陶瓷还没有统一的判定 标准。因此可以基于材料基因工程理念，发展高性 能 HETMCC 材料的设计理论和判据, 缩短高性能 HETMCC 材料的研发周期, 同时降低材料的制备 成本。

2)陶瓷粉体是制备 HETMCC 的原料, 开发出高 品质 HETMCC 粉体显得至关重要。目前 HETMCC 受烧结技术的限制，获得的陶瓷块体通常含有微气 孔，因此，亟需发展新的烧结技术。

3)受到 HEA 设计思想的启发, 高熵化的陶瓷由 于鸡尾酒效应的影响, 性能发生突变。通过对 HETMCC 陶瓷成分设计以及结构的调控，可以开发 出高强高韧的 HETMCC 陶瓷材料。 
4)目前 HETMCC 的研究仍集中在等摩尔比材 料, 虽然此时的构型熵最大, 但不一定是材料综合 性能最优的组成。如前所述, 高熵材料为我们提供 了多元相图中间广阔区域, 为新材料设计提供广泛 的空间。未来还需要对非等摩尔比的 HETMCC 组 成开展研究, 优化材料的综合性能。

5)相比于单组元碳化物陶瓷, HETMCC 的出现 较大程度地提高了碳化物陶瓷的抗氧化性。但是由 于阳离子数目多且存在选择性氧化, HETMCC 的氧 化机理还存在争议, 对于氧化的扩散控制过程是氧 向内扩散还是金属元素向外扩散需要进一步开展研 究, 为 HETMCC 抗氧化性能调控提供依据。

6) 烧蚀性能是 UHTCs 的核心指标之一, HETMCC 应用于超高温防热材料, 需要开展抗烧蚀 性能研究。到目前为止, 还没有关于 HETMCC 抗烧 蚀性能研究的报道, HETMCC 烧蚀机理也是未来 HETMCC 重点研究方向。

7) HEA 的高温稳定性是其应用的一个关键因 素, 同样 HETMCC 的高温稳定性也是需要关注的 问题，在更高温度下，单相 HETMCC 是否会发生局 部析出, 形成非化学计量比的第二相, 还有待探讨。

随着研究的不断深入, HETMCC 已经表现出许 多优异的性能。相信随着对 HETMCC 成分设计理 论的不断完善以及对新型制备技术的开发, HETMCC 将会有更广泛的应用空间。

\section{参考文献:}

[1] MIRACLE D B, SENKOV O N. A critical review of high entropy alloys and related concepts. Acta Mater., 2017, 122: 448-511.

[2] YEH J W, CHEN S K, LIN S J, et al. Nanostructured highentropy alloys with multiple principal elements: novel alloy design concepts and outcomes. Adv. Eng. Mater., 2004, 6(5): 299-303.

[3] KUCZA W, DABROWA, J, CIESLAK G, et al. Studies of "sluggish diffusion" effect in Co-Cr-Fe-Mn-Ni, Co-Cr-Fe-Ni and Co-Fe-Mn-Ni high entropy alloys; determination of tracer diffusivities by combinatorial approach. J. Alloys Compd., 2018, 731: 920-928.

[4] UTT D, STUKOWSKI A, ALBE K. Grain boundary structure and mobility in high-entropy alloys: a comparative molecular dynamics study on a 11 symmetrical tilt grain boundary in face-centered cubic CuNiCoFe. Acta Mater., 2020, 186: 11-19.

[5] GLUdovatz B, HOHENWARTER A, CATOOR D, et al. A fracture-resistant high-entropy alloy for cryogenic applications. Science, 2014, 345(6201): 1153-1158.

[6] LI Z M, PRADEEP K G, DENG Y, et al. Metastable high-entropy dual-phase alloys overcome the strength-ductility trade-off. Nature, 2016, 534(7606): 227-230.

[7] ZHANG R Z, REECE M J. Review of high entropy ceramics: design, synthesis, structure and properties. J. Mater. Chem. A, 2019, 7(39): 22148-22162.

[8] MURTY B S, YEH J W, RANGANATHAN S, et al. High-entropy Alloys. United Kingdom: Butterworth-Heinemann, 2019: 165-176.
[9] OSES C, TOHER C, CURTAROLO S. High-entropy ceramics. Nat. Rev. Mater., 2020, 5(4): 295-309.

[10] LAL M S, SUNDARA R. High entropy oxides-a cost-effective catalyst for the growth of high yield carbon nanotubes and their energy applications. ACS Appl. Mater. Inter., 2019, 11(34): 30846-30857.

[11] SARKAR A, VELASCO L, WANG D, et al. High entropy oxides for reversible energy storage. Nat. Commun., 2018, 9(1): 3400.

[12] BERARDAN D, FRANGER S, DRAGOE D, et al. Colossal dielectric constant in high entropy oxides. Phys. Status Solidi-R, 2016, 10(4): 328-333.

[13] BIESUZ M, SPIRIDIGLIOZZI L, DELLAGLi G, et al. Synthesis and sintering of $(\mathrm{Mg}, \mathrm{Co}, \mathrm{Ni}, \mathrm{Cu}, \mathrm{Zn}) \mathrm{O}$ entropy-stabilized oxides obtained by wet chemical methods. J. Mater. Sci., 2018, 53(11): 8074-8085.

[14] OKEJIRI F, ZHANG Z H, LIU J X, et al. Room-temperature synthesis of high-entropy perovskite oxide nanoparticle catalysts via ultrasonication-based method. ChemSusChem, 2020, 13(1): $111-115$.

[15] DEMIRSKYI D, BORODIANSKA H, SUZUKI T S, et al. Hightemperature flexural strength performance of ternary high-entropy carbide consolidated via spark plasma sintering of $\mathrm{TaC}, \mathrm{ZrC}$ and NbC. Scripta Mater., 2019, 164: 12-16.

[16] LI F, BAO W C, SUN S K, et al. Synthesis of single-phase metal oxycarbonitride ceramics. Scripta Mater., 2020, 176: 17-22.

[17] KUMAR A, GUPTA M. An insight into evolution of light weight high entropy alloys: a review. Metals Basel, 2016, 6(9): 199.

[18] ROST C M, SACHET E, BORMAN T, et al. Entropy-stabilized oxides. Nat. Commun., 2015, 6(1): 8485.

[19] WEI X F, QIN Y, LIU J X, et al. Gradient microstructure development and grain growth inhibition in high-entropy carbide ceramics prepared by reactive spark plasma sintering. J. Eur. Ceram. Soc., 2020, 40(4): 935-941.

[20] LI F, LU Y, WANG X G, et al. Liquid precursor-derived highentropy carbide nanopowders. Ceram. Int., 2019, 45(7): 22437-22441.

[21] WEI X F, LIU J X, LI F, et al. High entropy carbide ceramics from different starting materials. J. Eur. Ceram. Soc., 2019, 39(10): 2989-2994.

[22] DUSZA J, SVEC P, GIRMAN V, et al. Microstructure of (Hf-Ta-Zr-Nb)C high-entropy carbide at micro and nano/atomic level. J. Eur. Ceram. Soc., 2018, 38(12): 4303-4307.

[23] ZHOU J Y, ZHANG J Y, ZHANG F, et al. High-entropy carbide: a novel class of multicomponent ceramics. Ceram. Int., 2018, 44(17): 22014-22018.

[24] JIANG S C, HU T, GILD J, et al. A new class of high-entropy perovskite oxides. Scripta Mater., 2018, 142:116-120.

[25] ZHAO $\mathrm{Z}$ F, CHEN $\mathrm{H}$, XIANG $\mathrm{H} \mathrm{M}$, et al. $\left(\mathrm{Y}_{0.25} \mathrm{Yb}_{0.25} \mathrm{Er}_{0.25} \mathrm{Lu}_{0.25}\right)_{2}\left(\mathrm{Zr}_{0.5} \mathrm{Hf}_{0.5}\right)_{2} \mathrm{O}_{7}:$ a defective fluorite structured high entropy ceramic with low thermal conductivity and close thermal expansion coefficient to $\mathrm{Al}_{2} \mathrm{O}_{3}$. J. Mater. Sci. Technol., 2020, 39: 167-172.

[26] DABROWA J, STYGAR M, MIKULA A, et al. Synthesis and microstructure of the $\left(\mathrm{Co}, \mathrm{Cr}, \mathrm{Fe}, \mathrm{Mn}, \mathrm{Ni}_{3}\right)_{3} \mathrm{O}_{4}$ high entropy oxide characterized by spinel structure. Mater. Lett., 2018, 216: 32-36.

[27] ZHAO Z F, CHEN H, XIANG H M, et al. $\left(\mathrm{La}_{0.2} \mathrm{Ce}_{0.2} \mathrm{Nd}_{0.2} \mathrm{Sm}_{0.2} \mathrm{Eu}_{0.2}\right) \mathrm{PO}_{4}$ : a high-entropy rare-earth phosphate monazite ceramic with low thermal conductivity and good compatibility with $\mathrm{Al}_{2} \mathrm{O}_{3}$. J. Mater. Sci. Technol., 2020, 38: 80-85.

[28] GILD J, KAUFMANN K, Vecchio K, et al. Reactive flash spark plasma sintering of high-entropy ultrahigh temperature ceramics. Scripta. Mater., 2019, 170: 106-110.

[29] YAN J L, LIU F S, MA G H, et al. Suppression of the lattice thermal conductivity in NbFeSb-based half-Heusler thermoelectric 
materials through high entropy effects. Scripta Mater., 2018, 157: 129-134.

[30] GILD J, BRAUN J L, KAUFMANN K, et al. A high-entropy silicide: $\left(\mathrm{Mo}_{0.2} \mathrm{Nb}_{0.2} \mathrm{Ta}_{0.2} \mathrm{Ti}_{0.2} \mathrm{~W}_{0.2}\right) \mathrm{Si}_{2}$. J. Mater., 2019, 5(3): 337-343.

[31] SARKER P, HARRINGTON T, TOHER C, et al. High-entropy high-hardness metal carbides discovered by entropy descriptors. Nat. Commun., 2018, 9(1): 4980.

[32] SARKAR A, VELASCO L, WANG D, et al. High entropy oxides for reversible energy storage. Nat. Commun., 2018, 9(1): 3400.

[33] WANG H X, CAO Y J, LIU W, et al. Oxidation behavior of $\left(\mathrm{Hf}_{0.2} \mathrm{Ta}_{0.2} \mathrm{Zr}_{0.2} \mathrm{Ti}_{0.2} \mathrm{Nb}_{0.2}\right) \mathrm{C}-x \mathrm{SiC}$ ceramics at high temperature. Ceram. Int., 2020, 46(8): 11160-11168.

[34] TAN Y Q, CHEN C, Li S G, et al. Oxidation behaviours of high-entropy transition metal carbides in $1200{ }^{\circ} \mathrm{C}$ water vapor. $J$. Alloys Compd., 2020, 816: 152523.

[35] REN K, WANG Q K, SHAO G, et al. Multicomponent highentropy zirconates with comprehensive properties for advanced thermal barrier coating. Scripta Mater., 2020, 178: 382-386.

[36] DONG Y, REN K, LU Y H, et al. High-entropy environmental barrier coating for the ceramic matrix composites. J. Eur. Ceram. Soc., 2018, 39(7): 2574-2579.

[37] TENG Z, ZHU L N, TAN Y Q, et al. Synthesis and structures of high-entropy pyrochlore oxides. J. Eur. Ceram. Soc., 2020, 40(4): 1639-1643.

[38] ZHAO Z F, XIANG $\mathrm{H}$ M, DAI $\mathrm{F}$, et al. $\left(\mathrm{La}_{0.2} \mathrm{Ce}_{0.2} \mathrm{Nd}_{0.2} \mathrm{Sm}_{0.2} \mathrm{Eu}_{0.2}\right)_{2} \mathrm{Zr}_{2} \mathrm{O}_{7}$ : A novel high-entropy ceramic with low thermal conductivity and sluggish grain growth rate. $J$. Mater. Sci. Technol., 2019, 35(11): 2647-2651.

[39] SAVINO R, FUMO M D S, PATERNA D, et al. Aerothermodynamic study of UHTC-based thermal protection systems. Aerosp. Sci. Technol., 2005, 9(2): 151-160.

[40] OPRKA M M, TALMY I G, ZAYKOSKI J A. Oxidation-based materials selection for $2000{ }^{\circ} \mathrm{C}+$ hypersonic aerosurfaces: theoretical considerations and historical experience. J. Mater. Sci., 2004, 39(19): 5887-5904.

[41] KUBOTA Y, YANO M, INOUE R, et al. Oxidation behavior of $\mathrm{ZrB}_{2}-\mathrm{SiC}-\mathrm{ZrC}$ in oxygen-hydrogen torch environment. J. Eur. Ceram. Soc., 2017, 38(4): 1095-1102.

[42] RAMA RAO G A, VENUGOPAL V. Kinetics and mechanism of the oxidation of ZrC. J. Alloys Compd., 1994, 206(2): 237-242.

[43] VOITOVICH R F, PUGACH E A. High-temperature oxidation of ZrC and HfC. Powder Metall. Met. C, 1973, 12(11): 916-921.

[44] CHEN L Y, GU Y L, SHI L, et al. Synthesis and oxidation of nanocrystalline $\mathrm{HfB}_{2}$. J. Alloys Compd., 2004, 368(1): 353-356.

[45] SHIMADA S. Interfacial reaction on oxidation of carbides with formation of carbon. Solid State Ionics, 2001, 141: 99-104.

[46] PARTHASARATHY T A, RAPP R A, OPEKA M M, et al. A model for the oxidation of $\mathrm{ZrB}_{2}, \mathrm{HfB}_{2}$ and $\mathrm{TiB}_{2}$. Acta Mater., 2007, 55(17): 5999-6010.

[47] PARTHASARATHY T A, RAPP R A, OPEKA M M, et al. Effect of phase change and oxygen permeability in oxide scales on oxidation kinetics of $\mathrm{ZrB}_{2}$ and $\mathrm{HfB}_{2}$. J. Am. Ceram. Soc., 2009, 92(5): 1079-1086.

[48] JING Y, YUAN H B, LIAN Z S. Microstructure and mechanical properties of $\mathrm{ZrB}_{2}-\mathrm{HfC}$ ceramics influenced by $\mathrm{HfC}$ addition. Materials, 2018, 11(10): 2046.

[49] MALLIK M, RAY K K, MITRA R. Oxidation behavior of hot pressed $\mathrm{ZrB}_{2}-\mathrm{SiC}$ and $\mathrm{HfB}_{2}-\mathrm{SiC}$ composites. J. Eur. Ceram. Soc., 2011, 31(1): 199-215.

[50] TRIPP W C, GRAHAM H C. Thermogravimetric study of oxidation of $\mathrm{ZrB}_{2}$ in temperature range of $800{ }^{\circ} \mathrm{C}$ to $1500{ }^{\circ} \mathrm{C}$. J. Electrochem. Soc., 1971, 118(7): 1195-1199.
[51] FAHRENHOLTZ W G. The $\mathrm{ZrB}_{2}$ volatility diagram. J. Am. Ceram. Soc., 2005, 88(12): 3509-3512.

[52] FAHRENHOLTZ W G. Thermodynamic analysis of $\mathrm{ZrB}_{2}-\mathrm{SiC}$ oxidation: formation of a SiC-depleted region. J. Am. Ceram. Soc., 2007, 90(1): 143-148.

[53] HU P, GUOLIN W, WANG Z. Oxidation mechanism and resistance of $\mathrm{ZrB}_{2}-\mathrm{SiC}$ composites. Corros. Sci., 2009, 51(11): 2724-2732.

[54] JACOBSON N S, MYERS D L. Active oxidation of SiC. Oxid. Met., 2011, 75(1): 1-25.

[55] JACOBSON N S, HARDER B, MYERS D L, et al. Oxidation transitions for SiC. Part I. Active-to-passive transitions. J. Am. Ceram. Soc., 2013, 96(3): 838-844.

[56] WANG Y G, LUO L, SUN J, et al. $\mathrm{ZrB}_{2}-\mathrm{SiC}(\mathrm{Al})$ ceramics with high resistance to oxidation at $1500{ }^{\circ} \mathrm{C}$. Corros. Sci., 2013, 74: $154-158$.

[57] HE J B, WANG Y G, LUO L, et al. Oxidation behaviour of $\mathrm{ZrB}_{2}-\mathrm{SiC}(\mathrm{Al} / \mathrm{Y})$ ceramics at $1700{ }^{\circ} \mathrm{C}$. J. Eur. Ceram. Soc., 2016, 36(15): 3769-3774.

[58] WANG Y G, MA B S, LI L L, et al. Oxidation behavior of ZrB 2 -SiC-TaC ceramics. J. Am. Ceram. Soc., 2012, 95(1): 374-378.

[59] TONG Z W, HE R J, CHENG T B, et al. High temperature oxidation behavior of $\mathrm{ZrB}_{2}-\mathrm{SiC}$ added $\mathrm{MoSi}_{2}$ ceramics. Ceram. Int., 2018, 44(17): 21076-21082.

[60] ZAPATASOLVAS E, JAYASEELAN D D, BROWN P, et al. Effect of $\mathrm{La}_{2} \mathrm{O}_{3}$ addition on long-term oxidation kinetics of $\mathrm{ZrB}_{2}-\mathrm{SiC}$ and $\mathrm{HfB}_{2}-\mathrm{SiC}$ ultra-high temperature ceramics. J. Eur. Ceram. Soc., 2014, 34(15): 3535-3548.

[61] GILD J, ZHANG Y Y, HARRINGTON T, et al. High-entropy metal diborides: a new class of high-entropy materials and a new type of ultrahigh temperature ceramics. Sci. Rep-UK, 2016, 6(1): 37946-37946.

[62] YE B L, WEN T Q, HUANG K H, et al. First-principles study, fabrication, and characterization of $\left(\mathrm{Hf}_{0.2} \mathrm{Zr}_{0.2} \mathrm{Ta}_{0.2} \mathrm{Nb}_{0.2} \mathrm{Ti}_{0.2}\right) \mathrm{C}$ highentropy ceramic. J. Am. Ceram. Soc., 2019, 102(7): 4344-4352.

[63] HOSKING F M. Sodium compatibility of refractory-metal alloytype 3041 stainless-steel joints. Int. J. Refract. Met. H., 1985, 64(7): S181-S190.

[64] WERNER E A. Introduction to the thermodynamics of materials. Mat. Sci. Eng., 2008, 494(1/2): 464.

[65] CAR R, PARRINELLO M. Unified approach for molecular dynamics and density-functional theory. Phys. Rev. Lett., 1985, 55(22): 2471-2474.

[66] LIU X J, WANG C P, GAO F, et al. Thermodynamic calculation of phase equilibria in the $\mathrm{Sn}-\mathrm{Ag}-\mathrm{Cu}-\mathrm{Ni}-\mathrm{Au}$ System. J. Electron. Mater., 2007, 36(11): 1429-1441.

[67] WANG C P, WANG J, GUO S H, et al. Experimental investigation and thermodynamic calculation of the phase equilibria in the Co-Mo-W system. Intermetallics, 2009, 17(8): 642-650.

[68] FENG R, GAO M C, LEE C, et al. Design of light-weight highentropy alloys. Entropy Switz., 2016, 18(9): 333-353.

[69] KIM J. Applicability of special quasi-random structure models in thermodynamic calculations using semi-empirical Debye-Grüneisen theory. J. Alloys Compd., 2015, 650: 564-571.

[70] VOAS B K, USHER T M, LIU X M, et al. Special quasirandom structures to study the $\left(\mathrm{K}_{0.5} \mathrm{Na}_{0.5}\right) \mathrm{NbO}_{3}$ random alloy. Phys. Rev. B, 2014, 90(2): 024105-1-6.

[71] SAHARA R, EMURA S, LI S, et al. First-principles study of electronic structures and stability of body-centered cubic Ti-Mo alloys by special quasirandom structures. Sci. Technol. Adv. Mat., 2014, 15(3): 035014-1-10.

[72] VITOS L, ABRIKOSOV I A, JOHANSSON B. Anisotropic lattice distortions in random alloys from first-principles theory. Phys. Rev. 
Lett., 2001, 87(15): 156401-1-4.

[73] ABRIKOSOV I A, JOHANSSON B. Applicability of the coherent- potential approximation in the theory of random alloys. Phys. Rev. B, 1998, 57(22): 14164-14173.

[74] YE B L, WEN T Q, NGUYEN M C, et al. First-principles study, fabrication and characterization of $\left(\mathrm{Zr}_{0.25} \mathrm{Nb}_{0.25} \mathrm{Ti}_{0.25} \mathrm{~V}_{0.25}\right) \mathrm{C}$ highentropy ceramics. Acta Mater., 2019, 170: 15-23.

[75] BRAIC V, VLADESCU A, BALACEANU M, et al. Nanostructured multi-element (TiZrNbHfTa)N and (TiZrNbHfTa)C hard coatings. Surf. Coat. Tech., 2012, 211: 117-121.

[76] LIN S Y, CHANG S Y, HUANG Y C, et al. Mechanical performance and nanoindenting deformation of (AlCrTaTiZr) $\mathrm{NC}_{y}$ multi-component coatings co-sputtered with bias. Surf. Coat. Tech., 2012, 206(24): 5096-5102.

[77] ZHOU J Y, ZHANG J Y, ZHANG F, et al. high-entropy carbide: a novel class of multicomponent ceramics. Ceram. Int., 2018, 44(17): 22014-22018.

[78] FENG L, FAHRENHOLTZ W G, HILMAS G E, et al. Synthesis of single-phase high-entropy carbide powders. Scripta Mater., 2019, 162(12): 90-93.

[79] YE B L, NING S S, LIU D, et al. One-step synthesis of coral-like high-entropy metal carbide powders. J. Am. Ceram. Soc., 102(10): 6372-6378.

[80] DU B, LIU H H, CHU Y H. Fabrication and characterization of polymer-derived high-entropy carbide ceramic powders. J. Am. Ceram. Soc., 2020, 103: 4063-4068.

[81] NING S S, WEN T Q, YE B L, et al. Low-temperature molten salt synthesis of high-entropy carbide nanopowders. J. Am. Ceram. Soc., 2020, 103(3): 2244-2251.

[82] JAGADEESH S, VISHNU D S M, KIM H K, et al. Facile electrochemical synthesis of nanoscale (TiNbTaZrHf)C high-entropy carbide powder. Angew. Chem. Int. Ed., 2020 59(29): 11830-11835.

[83] BRAIC M, BRAIC V, BALACEANU M, et al. Characteristics of (TiAlCrNbY)C films deposited by reactive magnetron sputtering. Surf. Coat. Tech., 2010, 204(12): 2010-2014.

[84] JHONG Y S, HUANG C W, LIN S J, et al. Effects of $\mathrm{CH}_{4}$ flow ratio on the structure and properties of reactively sputtered (CrNbSiTiZr) $\mathrm{C}_{x}$ coatings. Mater. Chem. Phys., 2017, 210: 348-352.

[85] BRAIC M, BALACEANU M, VLADESCU A, et al. Deposition and characterization of multi-principal-element (CuSiTiYZr)C coatings. Appl. Surf. Sci., 2013 284: 671-678.

[86] BRAIC V, PARAU A C, PANA I, et al. Effects of substrate temperature and carbon content on the structure and properties of (CrCuNbTiY)C multicomponent coatings. Surf. Coat. Tech., 2014 258: 996-1005.

[87] CSANADI T, CASTLE E G, REECE M J, et al. Strength enhancement and slip behaviour of high-entropy carbide grains during micro-compression. Sci. Rep-UK, 2019, 9(1): 10200.

[88] WANG C, YE Y, GUAN X, et al. An analysis of tribological performance on $\mathrm{Cr} / \mathrm{GLC}$ film coupling with $\mathrm{Si}_{3} \mathrm{~N}_{4}, \mathrm{SiC}, \mathrm{WC}$, $\mathrm{Al}_{2} \mathrm{O}_{3}$ and $\mathrm{ZrO}_{2}$ in seawater. Tribol. Int., 2016 96: 77-86.

[89] HARRINGTON T J, GILD J, SARKER P, et al. Phase stability and mechanical properties of novel high entropy transition metal carbides. Acta Mater., 2019 166: 271-280.

[90] WANG K, CHEN L, XU C G, et al. Microstructure and mechanical properties of (TiZrNbTaMo)C high-entropy ceramic. $J$. Mater. Sci. Technol., 2020, 39: 99-105.

[91] HAN X X, VLADIMIR G, RICHARD S, et al. Improved creep resistance of high entropy transition metal carbides. J. Eur. Ceram. Soc., 2020, 40(7): 2709-2715.

[92] YAN $\mathrm{X}$ L, CONSTANTIN $\mathrm{L}$, LU $\mathrm{Y} F$, et al. $\left(\mathrm{Hf}_{0.2} \mathrm{Zr}_{0.2} \mathrm{Ta}_{0.2} \mathrm{Nb}_{0.2} \mathrm{Ti}_{0.2}\right) \mathrm{C}$ high-entropy ceramics with low thermal conductivity. J. Am. Ceram. Soc., 2018, 101(10): 4486-4491.

[93] CHEN H, XIANG H M, DAI F Z, et al. Porous high entropy $\left(\mathrm{Zr}_{0.2} \mathrm{Hf}_{0.2} \mathrm{Ti}_{0.2} \mathrm{Nb}_{0.2} \mathrm{Ta}_{0.2}\right) \mathrm{B}_{2}$ : a novel strategy towards making ultrahigh temperature ceramics thermal insulating. J. Mater. Sci. Technol., 2019, 35(10): 2404-2408.

[94] CHEN H, XIANG H M, DAI F Z, et al. High porosity and low thermal conductivity high entropy $\left(\mathrm{Zr}_{0.2} \mathrm{Hf}_{0.2} \mathrm{Ti}_{0.2} \mathrm{Nb}_{0.2} \mathrm{Ta}_{0.2}\right) \mathrm{C}$. J. Mater. Sci. Technol., 2019, 35(8): 1700-1705.

[95] YE B L, WEN T Q, LIU D, et al. Oxidation behavior of $\left(\mathrm{Hf}_{0.2} \mathrm{Zr}_{0.2} \mathrm{Ta}_{0.2} \mathrm{Nb}_{0.2} \mathrm{Ti}_{0.2}\right) \mathrm{C}$ high-entropy ceramics at $1073-1473 \mathrm{~K}$ in air. Corros. Sci., 2019, 153: 327-332.

[96] YE B L, WEN T Q, CHU Y H. High-temperature oxidation behavior of $\left(\mathrm{Hf}_{0.2} \mathrm{Zr}_{0.2} \mathrm{Ta}_{0.2} \mathrm{Nb}_{0.2} \mathrm{Ti}_{0.2}\right) \mathrm{C}$ high-entropy ceramics in air. J. Am. Ceram. Soc., 2019, 103(1): 500-507.

[97] WANG H X, HAN X, LIU W, et al. Oxidation behavior of high-entropy carbide $\left(\mathrm{Hf}_{0.2} \mathrm{Ta}_{0.2} \mathrm{Er}_{0.2} \mathrm{Ti}_{0.2} \mathrm{Nb}_{0.2}\right) \mathrm{C}$ at $1400-1600{ }^{\circ} \mathrm{C}$. DOI:10.1016/j.ceramint.2020.12.201.

[98] BACKMAN L, GILD J, LUO J, et al. Theoretical predictions of preferential oxidation in refractory high entropy materials. Acta Mater., 2020, 197: 20-27.

[99] WANG H X, WANG S Y, CAO Y J, et al. Oxidation behaviors of $\left(\mathrm{Hf}_{0.25} \mathrm{Zr}_{0.25} \mathrm{Ta}_{0.25} \mathrm{Nb}_{0.25}\right) \mathrm{C}$ and $\left(\mathrm{Hf}_{0.25} \mathrm{Zr}_{0.25} \mathrm{Ta}_{0.25} \mathrm{Nb}_{0.25}\right) \mathrm{C}-\mathrm{SiC}$ at 1300-1500 ㄷ. J. Mater. Sci. Technol., 2021, 60: 147-155.

[100] BRAIC V, BALACEANU M, BRAIC M, et al. Characterization of multi-principal-element (TiZrNbHfTa)N and (TiZrNbHfTa)C coatings for biomedical applications. J. Mech. Behav. Biomed., 2019, 10: 197-205.

[101] WANG F, YAN X L, WANG T Y, et al. Irradiation damage in $\left(\mathrm{Zr}_{0.25} \mathrm{Ta}_{0.25} \mathrm{Nb}_{0.25} \mathrm{Ti}_{0.25}\right) \mathrm{C}$ high-entropy carbide ceramics. Acta Mater., 2020, 195: 739-749. 